\title{
Search for New Physics with radiative B decays in Belle II
}

\section{Sviatoslav BILOKIN* on behalf of Belle II collaboration}

Institut Pluridisciplinaire Hubert CURIEN, Strasbourg, France

E-mail: sviatoslav.bilokineiphc.cnrs.fr

\begin{abstract}
This article presents a detailed analysis of $B^{0} \rightarrow K_{S}^{0} \pi^{+} \pi^{-} \gamma$ channel based on a full Monte-Carlo simulation of the Belle II experiment. This channel belongs to $b \rightarrow s \gamma$ penguin processes, which serve as sensitive probes of New Physics. Within the Standard Model the time-dependent $C P$ violation is expected to be small in $b \rightarrow s \gamma$ processes due to the chiral structure of the SM. Therefore, any significant $C P$ asymmetry in the $B^{0} \rightarrow K_{S}^{0} \pi^{+} \pi^{-} \gamma$ decay suggests a contribution of physics beyond the Standard Model.

The precision achieved by previous $B$-Factories, BaBar and Belle, on the $C P$ violating parameters in the $B^{0} \rightarrow K_{S}^{0} \pi^{+} \pi^{-} \gamma$ channel was statistically limited. This motivates a new measurement using the high integrated luminosity delivered by SuperKEKB and Belle II detector.

The statistical precision reached with the full Belle II dataset is expected to be $\delta S \approx 0.046$ and it will be comparable to the systematic uncertainty.
\end{abstract}

An Alpine LHC Physics Summit (ALPS2018)

15-20 April, 2018

Obergurgl, Austria

${ }^{*}$ Speaker. 


\section{Introduction}

In the Standard Model (SM) $b \rightarrow s \gamma$ transitions are forbidden at tree level and they are possible only through a quantum loop, see Fig. 1. Due to the chiral structure of the Standard Model the radiated photon is predominantly left-handed for $b$ and right-handed for $\bar{b}$ quarks. This makes $B-\bar{B}$ interference less probable and leads to a suppression $\left(\propto m_{s} / m_{b}\right)$ of the time-dependent $C P$ violation asymmetry. Some new physics scenarii [1] feature a different chiral structure and thus may lead to deviations from the SM expectation by introducing different photon polarizations in the transition. These New Physics models can be probed via experimental measurements of time-dependent CP violation (TDCPV) parameters at $B$-Factories.

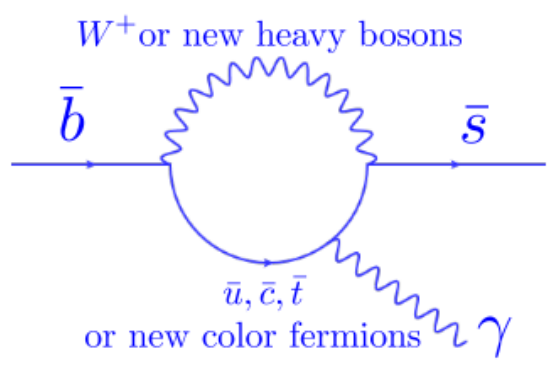

Figure 1: Feynman diagram of $b \rightarrow s \gamma$ transitions.

TDCPV analyses at $B$-Factories use $\Upsilon(4 S)$ to neutral $B$ meson decays, where the signal neutral $B$ meson is a $C P$ eigenstate and its companion neutral $B$ meson $(\operatorname{tag} B)$ is a flavor eigenstate used to infer the signal B flavor [2]. The mixing induced $C P$ violation is studied using the difference between the two $B$ meson decay time $(\Delta t)$. The $\Delta t$ distribution can be parametrized as:

$$
\mathscr{P}(\Delta t)=\frac{e^{-|\Delta t| / \tau_{B^{0}}}}{4 \tau_{B^{0}}}\{1+q \cdot[S \cdot \sin (\Delta m \Delta t)+A \cdot \cos (\Delta m \Delta t)]\},
$$

where $\tau_{B^{0}}$ is the $B$ proper decay time, $\Delta m$ is the mass difference between the two $B$ mass eigenstates, $q=+1(-1)$ is the b-flavor charge at a time of $\operatorname{tag} B$ decay, $A$ and $S$ are direct and mixing induced $C P$ violating parameters, respectively. Some experimental effects may impact the Eq. 1.1, such as possible $b$-flavor misidentification (mistag), experimental $\Delta t$ resolution and contributions of irreducible background.

One of the most powerful $b \rightarrow s \gamma$ channels is the $B^{0} \rightarrow K_{S}^{0} \pi^{+} \pi^{-} \gamma$ process, because of its significant branching fraction and easily analyzable final state composition. The signal $B$ meson decays to $X_{s d} \gamma$, where $X_{s d}$ denotes neutral K-resonances, like $K_{1}^{0}(1270)$ and $K^{* 0}(1410)$, which account for the largest contributions to the $K_{S}^{0} \pi^{ \pm} \pi^{\mp}$ final state. Several intermediate states, like $K^{* \pm} \pi^{\mp}$ are non- $C P$ eigenstates may dilute final TDCPV sensitivity [3]. This process has been measured previously by Belle [4] and BaBar [5] experiments, however, their results were statistically limited, see Table 1. This fact motivates further exploration of the $b \rightarrow s \gamma$ transitions using the expected larger dataset, which will be provided by the Belle II experiment at SuperKEKB collider.

\subsection{Belle II and SuperKEKB}

The Belle II experiment, successor of Belle, is designed for new physics searches via precision 


\begin{tabular}{c|c|c} 
& $B \bar{B}$ pairs analyzed & Measurement \\
\hline Belle & $467 \times 10^{6}$ & $\begin{array}{l}S=0.09 \pm 0.27_{-0.07}^{+0.04} \\
A=0.05 \pm 0.18 \pm 0.06\end{array}$ \\
\hline BaBar & $535 \times 10^{6}$ & $\begin{array}{l}S=0.14 \pm 0.25 \pm 0.03 \\
C=-0.39 \pm 0.20 \pm_{-0.02}^{+0.03}\end{array}$
\end{tabular}

Table 1: Results for TDCPV measurement with $B^{0} \rightarrow K_{S}^{0} \pi^{+} \pi^{-} \gamma$ channel by Belle [4] and BaBar [5] experiments.

measurements of $e^{+} e^{-}$collisions, and in particular of the B-meson decays. The first collisions has started in 2018 by the SuperKEKB collider with the full Belle II detector, but without the inner vertex detector installed. These first data runs are used for the detector commissioning and beam induced background studies. The final goal for the Belle II experiment is to collect $50 \mathrm{ab}^{-1}$ of integrated luminosity by 2025 .

SuperKEKB collides a $4 \mathrm{GeV}$ positron beam with a $7 \mathrm{GeV}$ electron beam, producing among others a $\Upsilon(4 S)$ resonance with a Lorentz boost of $\beta \gamma=0.28$. The main innovation concerning the accelerator part is the nano-beam scheme, which implies $60 \mathrm{~nm}$ vertical beam size at the interaction point. This upgrade along with many other accelerator improvements allow to target a very high instantaneous luminosity of $\mathscr{L}=8 \times 10^{35} \mathrm{~cm}^{-2} \mathrm{~s}^{-1}$. However, this achievement comes at a price of increased induced beam background impacting all Belle II subdetectors.

The Belle II detector inherits from its predecessor Belle and has been upgrade in accordance with the lower boost and higher beam induced background. It is designed as an asymmetric generalpurpose particle detector and it is composed of the following concentric devices:

- Innermost vertex detector consist of 2 layers of silicon pixels (PXD) and 4 double sided layers of silicon strips (SVD);

- Central tracking device is a small cell drift chamber (CDC);

- Time-Of-Propagation (TOP) and Aerogel Ring Imaging Cherenkov (ARICH) serve as particle identification (PID) devices;

- Electromagnetic calorimeter (ECL) equipped with CsI(Tl) crystals and photodiodes readout;

- Superconducting solenoid, which provides a $1.5 \mathrm{~T}$ magnetic field;

- Outermost $K_{L}^{0}$ and $\mu^{ \pm}$detector (KLM).

A detailed description of the Belle II experiment and SuperKEKB collider can be found in [6].

\section{Time-dependent $C P$ analysis of radiative decays}

The workflow of the $B^{0} \rightarrow K_{S}^{0} \pi^{+} \pi^{-} \gamma$ TDCPV analysis consist in the following steps:

- Signal final state selection, reconstruction and rejection background processes;

- Estimation of the $B \bar{B}^{0}$ flavor $q$ and the proper decay time difference $\Delta t$; 
- Construction of a fit model including mistag rate and $\Delta t$ resolution function and adjustment to the available dataset;

To reconstruct the signal $B^{0} \rightarrow K_{S}^{0} \pi^{+} \pi^{-} \gamma$ decay, we select a high energy photon with $E>$ $1.4 \mathrm{GeV}$. In order to reduce the contribution of photons coming from $\pi^{0} \rightarrow \gamma \gamma$ and $\eta \rightarrow \gamma \gamma$ decays we reject photon candidates using multi-variate analysis (MVA) technique. The neutral kaon $K_{S}^{0}$ is reconstructed combining two oppositely charged pions that have an invariant mass within $\pm 11 \mathrm{MeV}$ of the nominal $K_{S}^{0}$ mass. The reconstructed vertex of the $K_{S}^{0}$ candidate is required to be $>5 \sigma_{K_{S}^{0}}$ away from the interaction point (IP), where $\sigma_{K_{S}^{0}}$ is the uncertainty of the reconstructed $K_{S}^{0}$ flight distance. All charged pions in the signal final state are required to have a $\pi^{ \pm}$PID likeness probability of more than $5 \%$. The invariant mass of two charged pions, which do not originate from $K_{S}^{0}$, are required to be between 0.6 and $0.8 \mathrm{GeV}$ to enrich the signal sample with $C P$ eigenstate candidates.

Beam-constrained variables $m_{b c}=\sqrt{\left(E_{\text {beam }}^{\text {c.m.s. }}\right)^{2}-\left(p_{B}^{\text {c.m.s. }}\right)^{2}}$ and $\Delta E=E_{B}^{\text {c.m.s. }}-E_{\text {beam }}^{\text {c.m.s. }}$ are used for background rejection, where $E_{\text {beam }}^{\text {c.m.s. }}$ is the beam energy, and $E_{B}^{\text {c.m.s. }}$ and $p_{B}^{\text {c.m.s. }}$ are the energy and momentum of the signal $B$ candidate in the center-of-mass (c.m.s.) system. The signal $B$ candidate is required to have $5.2<m_{b c}<5.3$ and $|\Delta E|<0.2$.

To reject the continuum background processes originating from the $e^{+} e^{-} \rightarrow u \bar{u}, d \bar{d}, s \bar{s}, c \bar{c}$ processes an MVA likeness is constructed based on event shape variables, like Fox-Wolfram moments and CLEO Cones. This MVA likeness is referred as Continuum Suppression MVA (CSMVA).

The $b$-flavor of the $\operatorname{tag} B$ is reconstructed using the properties of particles that are not associated with the signal $B$ and applying MVA technique. The flavor-tag algorithm in Belle II software outputs the flavor quality factor $r$ times the reconstructed $b$-flavor charge $q$ from Eq. 1.1. Using the flavor-tag output and the generated $b$-flavor values, the mistag rate distribution is extracted, which will be used to measure $C P$ violating parameters.

The proper decay time difference $\Delta t$ is calculated using the reconstructed signal $B$ and $\operatorname{tag} B$ vertices. To increase the $\Delta t$ reconstruction quality we apply a cut on its uncertainty $\sigma_{\Delta t}<2.5 \mathrm{ps}$. The $\Delta t$ residual is defined as $\Delta t_{\text {rec }}-\Delta t_{\text {gen }}$ using simulated data, where $\Delta t_{g e n}$ and $\Delta t_{\text {rec }}$ are the generated and reconstructed $\Delta t$ values event-per-event, respectively. To obtain the resolution function, the $\Delta t$ residual distribution is fitted by a sum of three Gaussian distributions with a center at 0 and average width of $2.2 \mathrm{ps}$.

\section{Results}

In this work the TDCPV sensitivity is estimated using full simulation of Belle II experiment. The available size of simulated dataset correspond to $0.63 \mathrm{ab}^{-1}$ of integrated luminosity. The CSMVA is trained using a large independent signal Monte-Carlo sample. Values and statistical uncertainties of signal event rate and TDCPV parameters are extracted using an unbinned 4-dimentional maximum likelihood fit method to $m_{b c}, \Delta E, \Delta t$ and Continuum Suppression output. Results of the fit of an available full simulation dataset corresponding to $0.63 \mathrm{ab}^{-1}$ of integrated luminosity is shown in Fig. 2. Corresponding statistical uncertainties on TDCPV parameters are: $\delta A_{\text {stat }}=0.26$ and $\delta S_{\text {stat }}=0.37$. A detailed sensitivity study was done based on a Toy MC and the information obtained with full Belle II simulation, see Fig. 3. With the full expected dataset 

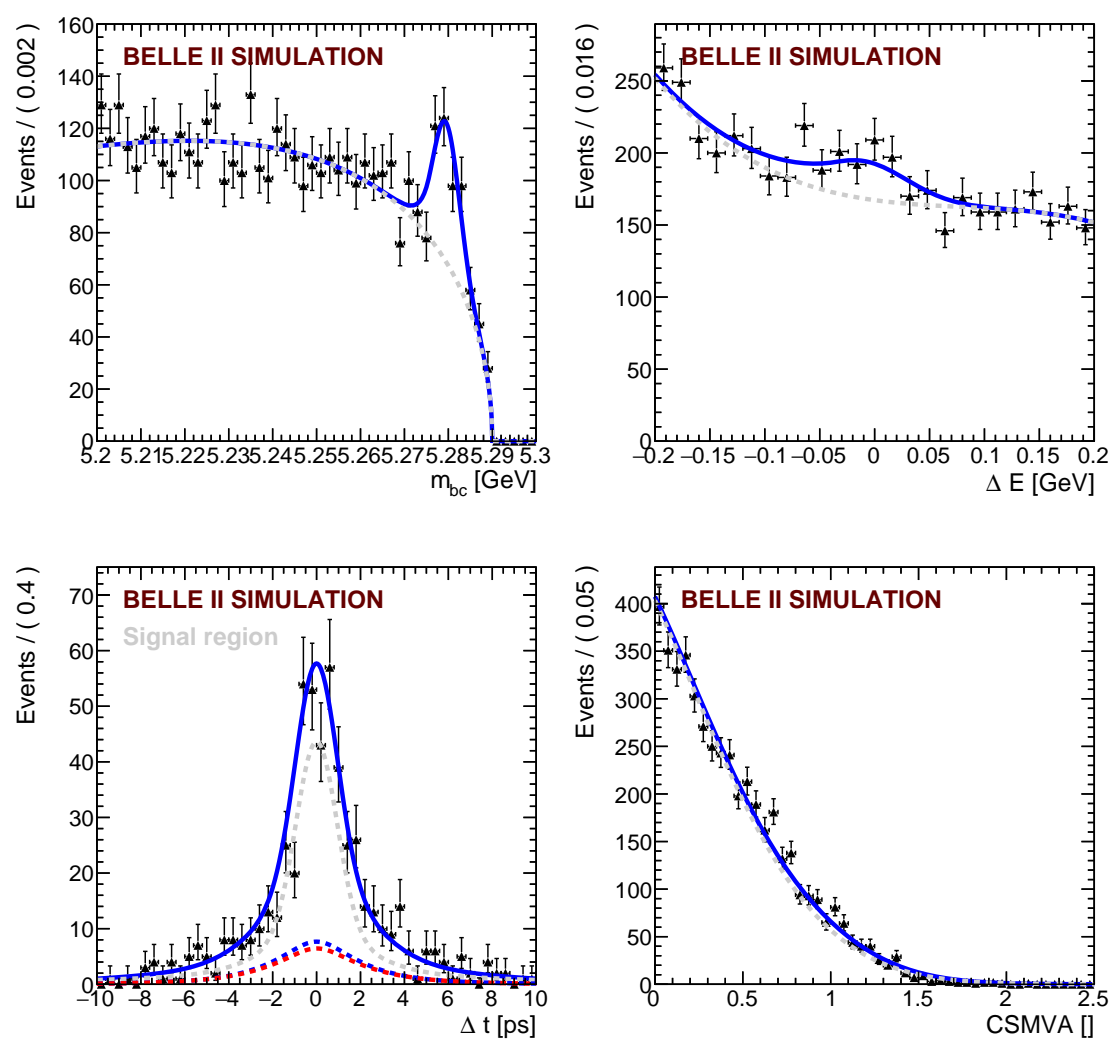

Figure 2: Distributions of the four main observables used in the measurement, from top left to bottom right: $m_{b c}, \Delta E, \Delta t$ and CSMVA. Points correspond to simulated signal and background events and the corresponding fit function projections depicted with a blue line.

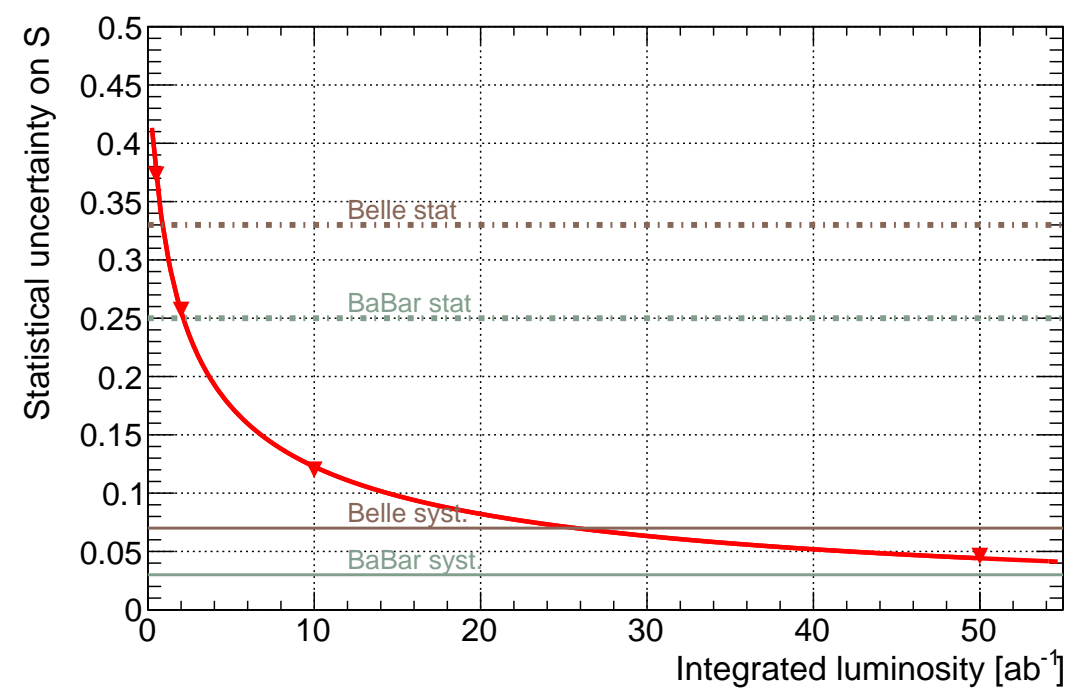

Figure 3: Belle II prospects for TDCPV measurement of $B^{0} \rightarrow K_{S}^{0} \pi^{+} \pi^{-} \gamma$ channel. Horizontal dotted and solid lines correspond respectively to statistical and systematic uncertainties obtained at the previous $B$ factories. 
of $50 \mathrm{ab}^{-1}$ the precision on the $S$ parameter measured with $B^{0} \rightarrow K_{S}^{0} \pi^{+} \pi^{-} \gamma$ decays will be on the order of $\delta S \approx 0.046$. This statistical uncertainty is compatible with the systematic uncertainties by Belle [4] and BaBar [5] analyses. Due to a rich structure of higher K-resonances decaying to $K_{S}^{0} \pi^{ \pm} \pi^{\mp}$ [3], presence of non-CP eigenstates in the fitted dataset may dilute the precision on $S$ parameter at the order of $20 \%$ [5].

\section{Conclusions}

This article describes a full simulation time-dependent $C P$ analysis of $B^{0} \rightarrow K_{S}^{0} \pi^{+} \pi^{-} \gamma$ channel, which belongs to $b \rightarrow s \gamma$ processes. Computed statistical precision on the $S$ parameter is $\delta S \approx 0.046$, comparable to the systematic uncertainties obtained at previous $B$-factories. The current status of the analysis indicates that the investigation of non-SM photon polarization through TDCPV measurement should be limited by systematics at the end of Belle II data taking. However, one should take this initial indication cautiously. Indeed, this results also depends on the beam induced background level, which is being carefully evaluated with the 2018 dataset registered by Belle II at SuperKEKB. Improvements in the analysis are also to be expected since the mentioned cut values and fit ranges have still to be optimized. Furthermore, another channel $B^{0} \rightarrow K_{S}^{0} \pi^{0} \gamma$ can bring additional insight into the photon polarization. The short term prospect regarding these channels is the analysis of the 2019 Belle II data campaign, which should bring similar statistics as the previous B-factories.

\section{References}

[1] D. Atwood, M. Gronau and A. Soni, Mixing-induced CP asymmetries in radiative B decays in and beyond the standard model, Phys. Rev. Lett. 79 (1997) 185.

[2] BELLE collaboration, H. Kakuno et al., Neutral B flavor tagging for the measurement of mixing induced CP violation at Belle, Nucl. Instrum. Meth. A533 (2004) 516 [hep-ex/ 0403022 ].

[3] S. Akar, E. Ben-Haim, J. Hebinger, E. Kou and F.-S. Yu, The time-dependent CP asymmetry in $B^{0} \rightarrow K_{\mathrm{res}} \gamma \rightarrow \pi^{+} \pi^{-} K_{S}^{0} \gamma$ decays, 1802.09433.

[4] Belle collaboration, J. Li et al., Time-dependent CP Asymmetries in $B^{0} \rightarrow K_{S}^{0} \rho^{0} \gamma$ Decays, Phys. Rev. Lett. 101 (2008) 251601 [0806.1980].

[5] BABAR collaboration, P. del Amo Sanchez et al., Time-dependent analysis of $B^{0} \rightarrow K_{S}^{0} \pi^{-} \pi^{+} \gamma$ decays and studies of the $K^{+} \pi^{-} \pi^{+}$system in $B^{+} \rightarrow K^{+} \pi^{-} \pi^{+} \gamma$ decays, Phys. Rev. D93 (2016) 052013 [1512.03579].

[6] BELLE-II collaboration, T. Abe et al., Belle II Technical Design Report, tech. rep., KEK, 2010. 